\title{
Bazedoxifene, a selective estrogen receptor modulator, reduces cerebral aneurysm rupture in Ovariectomized rats
}

Hidetsugu Maekawa ${ }^{1 *} \mathbb{D}$, Yoshiteru Tada', Kenji Yagi ${ }^{1}$, Takeshi Miyamoto ${ }^{1}$, Keiko T. Kitazato', Masaaki Korai ${ }^{1}$, Junichiro Satomi ${ }^{1}$, Tomoki Hashimoto ${ }^{2}$ and Shinji Nagahiro ${ }^{1}$

\begin{abstract}
Background: Estrogen deficiency is thought to be responsible for the higher frequency of aneurysmal subarachnoid hemorrhage in post- than premenopausal women. Estrogen replacement therapy appears to reduce this risk but is associated with significant side effects. We tested our hypothesis that bazedoxifene, a clinically used selective estrogen receptor (ER) modulator with fewer estrogenic side effects, reduces cerebral aneurysm rupture in a new model of ovariectomized rats.

Methods: Ten-week-old female Sprague-Dawley rats were subjected to ovariectomy, hemodynamic changes, and hypertension to induce aneurysms (ovariectomized aneurysm rats) and treated with vehicle or with 0.3 or $1.0 \mathrm{mg} / \mathrm{kg} /$ day bazedoxifene. They were compared with sham-ovariectomized rats subjected to hypertension and hemodynamic changes (HT rats). The vasoprotective effects of bazedoxifene and the mechanisms underlying its efficacy were analyzed.
\end{abstract}

Results: During 12 weeks of observation, the incidence of aneurysm rupture was $52 \%$ in ovariectomized rats. With no effect on the blood pressure, treatment with 0.3 or $1.0 \mathrm{mg} / \mathrm{kg} /$ day bazedoxifene lowered this rate to 11 and $17 \%$, almost the same as in $H T$ rats (17\%). In ovariectomized rats, the mRNA level of ERa, ERß, and the tissue inhibitor of metalloproteinase-2 was downregulated in the cerebral artery prone to rupture at 5 weeks after aneurysm induction; the mRNA level of interleukin-1 $\beta$ and the matrix metalloproteinase- 9 was upregulated. In HT rats, bazedoxifene restored the mRNA level of ERa and ER $\beta$ and decreased the level of interleukin-1 $\beta$ and matrix metalloproteinase-9. These findings suggest that bazedoxifene was protective against aneurysmal rupture by alleviating the vascular inflammation and degradation exacerbated by the decrease in ERa and ERß.

Conclusions: Our observation that bazedoxifene decreased the incidence of aneurysmal rupture in ovariectomized rats warrants further studies to validate this response in humans.

Keywords: Estrogen, Estrogen receptor, Intracranial aneurysm, Selective estrogen receptor modulator, Subarachnoid hemorrhage

\footnotetext{
* Correspondence: hidetsugumaekawa@yahoo.co.jp

${ }^{1}$ Department of Neurosurgery, Institute of Biomedical Sciences, Tokushima

University Graduate School, 3-18-15 Kuramoto-cho, Tokushima 770-8503,

Japan

Full list of author information is available at the end of the article
} 


\section{Background}

The rupture of cerebral aneurysms elicits devastating subarachnoid hemorrhage (SAH). Surgical and endovascular treatments can be preventive but are associated with inherent risks and complications [1]. Therefore, medical therapy with lower risks for complications is needed to prevent SAH.

The incidence of cerebral aneurysms and $\mathrm{SAH}$ is higher in post- than premenopausal women $[2,3]$. Postmenopausal estrogen deficiency is thought to play a key role in the pathophysiology of cerebral aneurysms. Our group established a cerebral aneurysm model in ovariectomized rats subjected to hemodynamic stress and hypertension [4]. The model mimics human postmenopausal hormonal conditions. Estrogen-deficient model rats had a higher incidence of cerebral aneurysms than male rats, and estrogen deficiency promoted endothelial damage and vascular inflammation [5-8]. Estrogen (17 $\beta$-estradiol) and an estrogen receptor $\beta$ (ER $\beta$ )-, but not an ER $\alpha$ agonist, reduced the incidence of cerebral aneurysm rupture in ovariectomized mice [9]. These findings suggest that ERrelated drugs may help to prevent SAH. In humans, postmenopausal estrogen replacement therapy reduced the risk of SAH [10-12] but increased the risk for breast and endometrial cancer, stroke, and venous thromboembolism [9, 13-15].

Bazedoxifene (BZA), a selective estrogen receptor modulator (SERM), is used to prevent postmenopausal osteoporosis. It has fewer estrogenic side effects than conjugated estrogen. SERM is a ligand of ER $\alpha$ and ER $\beta$; its characteristics are different from pure ER agonists and ER-antagonists because, depending on the target tissue, it exerts agonistic or antagonistic effects [16]. Its tissue-specific effects are thought to be attributable to its distinct affinity for each ER, a unique conformational change in ERs upon binding to SERM, and the distinct distribution of ER $\alpha$ and ER $\beta$ in the tissue [16, 17]. Therefore, we used our rat model to investigate whether BZA may represent a potential and safer prophylactic agent against cerebral aneurysm rupture in postmenopausal women.

We established a novel rat model of aneurysm rupture by modifying the carotid ligation procedure used in our original model in which ovariectomized rats were subjected to hemodynamic changes and hypertension without pharmacologically elicited degeneration of the arterial wall [18]. The pathological features of the experimental aneurysms in our ovariectomized hypertensive rats were those of human aneurysms. In the posterior cerebral artery (PCA) where ruptured aneurysms formed most frequently, the mRNA level of interleukin (IL)-1 $\beta$ was higher and the imbalance of matrix metalloproteinase (MMP)-9 and the level of the tissue inhibitor of metalloproteinase-2 (TIMP-2) were greater than at the bifurcation of the anterior cerebral artery-olfactory artery (ACA-OA) where no ruptured aneurysms were observed. We suggested that these molecules associated with inflammation and vascular degradation are at least partly responsible for aneurysmal rupture [18]. Here, we used our modified rat aneurysm model to test the hypothesis that BZA exerts protective effects against vascular inflammation and aneurysm rupture.

We now demonstrate that BZA reduced the incidence of aneurysmal rupture without affecting the blood pressure in ovariectomized aneurysm-model rats. Its restoration of the decreased expression of both ERs in the aneurysm-prone cerebral artery was associated with the reduction of pro-inflammatory cytokines and vascular degradation molecules. We also show that BZA attenuated the body weight gain seen under conditions of estrogen deficiency and hypertension. Our findings suggest BZA as a potential therapeutic agent for the prevention of SAH in postmenopausal women.

\section{Methods \\ Animals}

Sexually mature (10-week-old) [19] female Sprague-Dawley rats (220-250 g, $n=137$; Charles River Laboratories Japan Inc., Yokohama, Japan) were housed under specificpathogen-free conditions and controlled temperatures $\left(23 \pm 2{ }^{\circ} \mathrm{C}\right)$, humidity $(55 \pm 10 \%)$, and light (12-h light/dark cycle, 8 am-8 pm). Food and water were ad libitum.

\section{Cerebral aneurysm induction}

Cerebral aneurysms were induced by hypertension (renal artery ligation and a high-salt diet), increased hemodynamic changes (carotid artery ligation), and estrogen deficiency (bilateral ovariectomy) as described elsewhere [18]. We ligated the right external carotid and the pterygopalatine artery and the left common carotid artery. The rats were bilaterally ovariectomized immediately after carotid ligation and then fed a high-salt diet $(8 \%$ sodium chloride). Two weeks later, the bilateral posterior renal arteries were ligated.

\section{Experimental study 1}

To assess whether ovariectomy increases and BZA treatment reduces the incidence of cerebral aneurysm rupture in hypertensive ovariectomized rats, 84 rats were randomly assigned to three groups (each group $n=28$ ). One group was treated with vehicle (HT+OVX); the other two groups received 0.3 or $1.0 \mathrm{mg} / \mathrm{kg} /$ day BZA (BZA0.3, BZA1, respectively). Another set of 28 rats was sham-ovariectomized, subjected to hypertension, exposed to hemodynamic changes, and treated with vehicle (HT rats). BZA was kindly provided by Pfizer Inc. (New York, NY, USA). The BZA dose, determined based on an earlier study [20], was suspended in 5\% Arabic gum solution 
(BZA0.3 rats: $0.06 \mathrm{mg} / \mathrm{ml}$, BZA1 rats: $0.2 \mathrm{mg} / \mathrm{ml}$ ) and perorally administered once a day $(5 \mathrm{ml} / \mathrm{kg}$ for all solutions) for 12 weeks by blinded investigators. The $5 \%$ Arabic gum solution was also the vehicle. In our previous study, cerebral aneurysms started to rupture approximately 30 days after their induction [18]. The administration of BZA and of vehicle was started 2 weeks after renal artery ligation so that BZA would affect the rupture rather than the formation of aneurysms.

Aneurysm rupture was suspected when the rats exhibited neurologically abnormal behavior [21], developed a significant weight loss (> $30 \mathrm{~g} /$ day, approximately $10 \%$ of their body weight), or died. Rats with these findings were sacrificed immediately, and their brains were inspected to confirm SAH due to rupture of a cerebral aneurysm. Blinded investigators recorded the body weight daily to detect a sudden weight loss possibly indicative of aneurysmal rupture, assessed the clinical signs, and evaluated the brains. A representative ruptured aneurysm at the left PCA is shown in Additional file 1: Figure S1. All surviving rats were euthanized 12 weeks after aneurysm induction, and their brains were inspected for the presence of ruptured aneurysms. Data collection and analysis were performed in blinded fashion.

Of the 112 rats, we excluded 14 ( $5 \mathrm{HT}+\mathrm{OVX}, 1 \mathrm{BZA} 0.3$, 4 BZA1, and 4 HT rats) because there was no evidence of rupture, although aneurysmal rupture was suspected.

\section{Blood pressure measurements}

To measure the blood pressure, the rats were placed on a $37{ }^{\circ} \mathrm{C}$ hot plate (Nissin, Tokyo, Japan) and covered with a blanket. After they adapted to the environment, their systolic blood pressure was measured twice, and the mean value was recorded. Blood pressure measurements were obtained immediately before and 2 and 12 weeks after renal artery ligation by the tail-cuff, auto-pickup method (Softron, Tokyo, Japan).

\section{Experimental study 2}

To analyze the molecular mechanisms underlying the effects of ovariectomy and BZA on rat cerebral aneurysms, we performed quantitative real-time PCR (qRT-PCR) to assess the gene expression level and immunohistochemical staining to determine the protein expression level in the cerebral artery.

\section{qRT-PCR assay}

Another set of 21 rats was randomly divided into three equal groups of $\mathrm{HT}+\mathrm{OVX}$, BZA1, and HT rats. Bazedoxifene or vehicle was administered as in the "Experimental study 1" section. We did not prepare a BZA0.3 group because experimental study 1 had shown that the incidence of cerebral aneurysm rupture was not significantly different between BZA0.3 and BZA1 rats.
To avoid excluding rats that would develop aneurysmal rupture, we euthanized these rats at 5 weeks after renal ligation before their experimentally induced aneurysms had begun to rupture in experimental study 1 . We collected tissue samples from these 21 rats from the left proximal PCA (between the basilar bifurcation and the posterior communicating artery) where ruptured cerebral aneurysms had formed most frequently in $\mathrm{HT}+\mathrm{OVX}$ rats in experimental study 1 .

After transcardial perfusion with normal saline, we removed the left PCA. Total RNA was extracted with the MagNA Pure RNA isolation kit (Roche, Tokyo, Japan) and placed in a MagNA lyser (Roche). We used Transcriptor Universal cDNA Master (Roche) for the reverse transcription of total RNA to cDNA and a LightCycler 2.0 (Roche Diagnostics, Tokyo, Japan) for qRT-PCR. LightCycler FastStart DNA Master and SYBR Green I (Roche) were used for glyceraldehyde 3-phosphate dehydrogenase (GAPDH), ER $\alpha$, ER $\beta$, IL-1 $\beta$, IL-6, tumor necrosis factor- $\alpha$ (TNF- $\alpha)$, MMP-9, and TIMP-2. The primers were as follows: for $\mathrm{GAPDH}$, forward (F), $5^{\prime}-\mathrm{TAC}$ ACT GAG GAC CAG GTT G-3', reverse (R), 5'-CCC TGT TGC TGT AGC CAT A-3'; for ER $\alpha$, F, 5' -TGC ACC ATC GAT AAG AAC C-3', R, 5'-GTC TCC TGA AGT GCC CAT T-3'; for ER $\beta, F, 5^{\prime}$-CTG CAT GGC TGA GCG ACA A-3', R, 5' -AGA GAC TCA TGG GAC TCA GAT-3'; for IL-1 $\beta$, F, 5'-TGC AGG CTT CGA GAT GAA C-3', R, 5'-AGC TCA TGG AGA ATA CCA CTT G-3'; for IL-6, F, 5' -TCT CAG GGA GAT CTT GGA AAT G-3', R, 5' -TAG AAA CGG AAC TCC AGA C-3'; for TNF- $\alpha, F$, 5'-CCC AAC AAG GAG AAG T-3', R, 5'-CGC TTG GTG GTT TGC TAC-3'; for MMP-9, F, 5'-CCT GGA ACT CAC ACA ACG-3', R, 5'-GAG GTC ATA GGT CAC GTA GG -3'; and for TIMP-2, F, 5'-CCC TCT GTG ACT TTA TTG TGC-3', R, 5'-TGA TGC TCT CTG TGA CC-3'. The PCR conditions were $95{ }^{\circ} \mathrm{C}$ for $10 \mathrm{~min}$ followed by 40 cycles at $95^{\circ} \mathrm{C}$ for $10 \mathrm{~s}, 60{ }^{\circ} \mathrm{C}$ for $10 \mathrm{~s}$, and $72{ }^{\circ} \mathrm{C}$ for $8 \mathrm{~s}$. We subjected samples from each group to two independent qRT-PCR assays to determine the gene expression level. The results were quantified after normalization to the expression of GAPDH mRNA. To assess the effect of BZA on the uterus, we collected the uterus and measured its weight.

\section{Immunohistochemical staining}

The left proximal PCA from two $\mathrm{HT}+\mathrm{OVX}$ and two BZA1 rats was collected 5 weeks after renal ligation to confirm ER protein expression in the arterial wall. After perfusion with $4 \%$ paraformaldehyde, the left PCA was harvested, immersed in $4 \%$ paraformaldehyde $(24 \mathrm{~h}$ at $4{ }^{\circ} \mathrm{C}$ ), and successively dehydrated in 10,20 , and $30 \%$ sucrose. The arteries were rinsed with phosphate-buffered saline, embedded in OCT compound (Tissue-Tek Inc.), and cut into $5-\mu \mathrm{m}$ thick serial sections with a cryotome 
(CM 1850; Leica). After 30-min serum-free protein blockade (Dako, Carpinteria, CA, USA), primary antibodies against ER $\alpha$ and ER $\beta$ (Santa Cruz Biotechnology, CA, USA) diluted 1:100 with Canget signal immunostain (Toyobo, Osaka, Japan) were added for overnight incubation at $4{ }^{\circ} \mathrm{C}$. Sections not treated with the primary antibodies were the negative control. All sections were then incubated for $1 \mathrm{~h}$ at room temperature with fluorescein-conjugated secondary antibodies Alexa Fluor 594 (Molecular Probes, CA, USA) in Canget signal immunostain (1:800 dilution, Toyobo), mounted with Vectashield (Vector Laboratories, CA, USA), and examined under a fluorescence microscope (KEYENCE, BZ-X710, Osaka, Japan).

\section{Statistical analysis}

For sample-size calculation, we implemented $\alpha=0.05$ and $1-\beta=0.8$ with an effect size of 0.4 (literature-based) [9] using G*Power 3.1 (University of Düsseldorf, Düsseldorf, Germany), indicating that 25 samples per group were required. Expecting a loss of $10-15 \%$ of the rats, we used 28 animals per group in experimental study 1 . Statistical analyses were performed with SPSS 22 (IBM Armonk, $\mathrm{NY}$ ). Fisher's exact test was used to analyze the incidence of cerebral aneurysm rupture. Sequentially, obtained data (mean $\pm \mathrm{SD}$ ) were analyzed with analysis of variance (ANOVA) followed by Scheffe's test for multiple group comparisons of the mRNA level. Differences were considered statistically significant at $p<0.05$.

\section{Results}

BZA prevents aneurysmal rupture in rats

To determine whether estrogen deficiency increases while BZA decreases the incidence of cerebral aneurysm rupture in estrogen-deficient rats, we compared the incidence of rupture during 12 weeks of observation in hypertensive ovariectomized aneurysm rats treated with vehicle (HT +OVX), $0.3 \mathrm{mg} / \mathrm{kg}$ BZA (BZA0.3), or $1 \mathrm{mg} / \mathrm{kg} \mathrm{BZA}$ (BZA1) and in hypertensive sham-ovariectomized rats (HT rats) (for their preparation see the "Experimental study 1" section). The treatments were started 2 weeks after bilateral renal ligation and continued throughout the 12-week observation period.

As shown in Fig. 1a, HT+OVX rats manifested a significantly higher incidence of cerebral aneurysm rupture than HT rats (52 vs $17 \%, p<0.05)$. BZA treatment decreased the incidence of rupture (BZA0.3, $11 \%$; BZA1, $17 \%$; each $p<0.05$ vs $\mathrm{HT}+\mathrm{OVX}$ rats). Notably, the rate of aneurysmal rupture in BZA0.3 and BZA1 rats was similar to that in HT rats, suggesting that the effect of BZA was not dose-dependent. BZA did not affect the systolic blood pressure, which was elevated after renal ligation (Fig. 1b). These results suggest that estrogen deficiency promoted aneurysm rupture in the presence of hypertension, while BZA inhibited its effect in a blood pressure-independent manner. Most ruptured aneurysms had arisen in the PCA (64\%); 14\% each were found in the internal carotid or the anterior cerebral artery (14\%), and $9 \%$ in the middle cerebral artery.

Although there was no significant difference in the body weight at the time of randomization [HT, $251.8 \pm 16.2$ g; HT+OVX, $260.9 \pm 22.7$ g; BZA0.3, $261.0 \pm 16.4$ g; BZA1, $263.7 \pm 12.7 \mathrm{~g}$ (mean $\pm \mathrm{SD})], 12$ weeks after aneurysm induction, the body weight of $\mathrm{HT}+\mathrm{OVX}$ rats was significantly greater than that of HT rats $(381.4 \pm 27.6$ vs $300.2 \pm 18.8 \mathrm{~g}, p<0.05)$. The body weight of BZA1 was significantly lower than that of $\mathrm{HT}+\mathrm{OVX}$ rats $(263.7 \pm 12.7 \mathrm{~g}$ vs $335.3 \pm 17.3 \mathrm{~g})$; treatment with $0.3 \mathrm{mg} /$ $\mathrm{kg}$ BZA did not affect the body weight $(375.8 \pm 24.4 \mathrm{~g})$ (Fig. 1c). In addition, 5 weeks after aneurysm induction, the wet uterine weight of $\mathrm{HT}+\mathrm{OVX}$ rats was significantly lower than that of HT rats; it was similar in BZA1 and HT
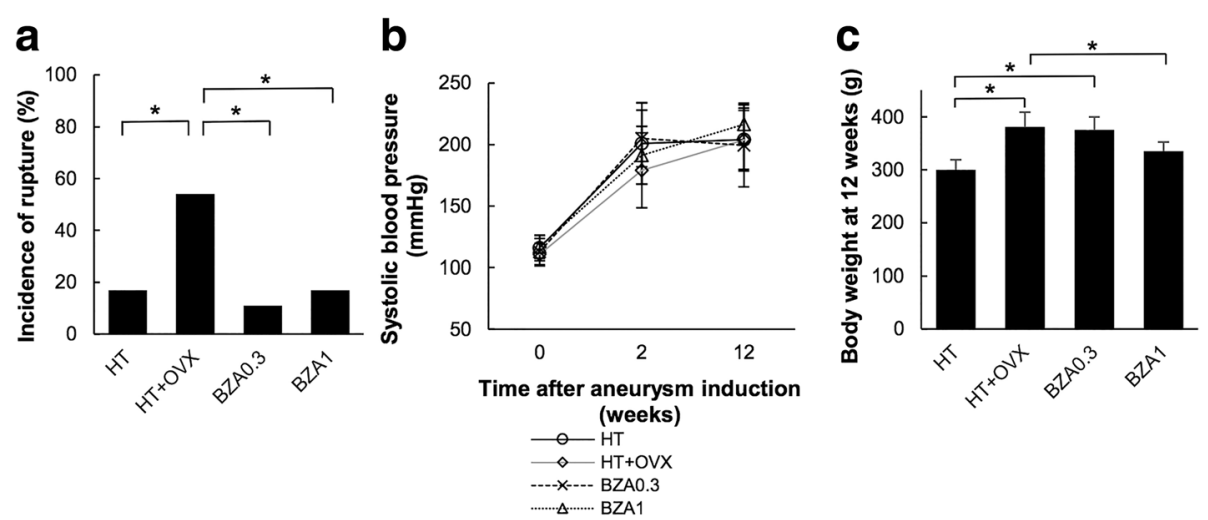

Fig. 1 a Incidence of aneurysm rupture within 12 weeks after aneurysm induction $\left({ }^{*} p<0.05\right.$, Fisher's exact test). b Sequential changes in the systolic blood pressure ( ${ }^{*} p<0.05$ vs sham, Scheffe's test). c The body weight at 12 weeks after aneurysm induction $\left({ }^{*} p<0.05\right.$, Scheffe's test). Data are the mean \pm SD. $n=23-27$ per group 
+OVX rats (HT, $440.5 \pm 48.1 \mathrm{mg} ; \mathrm{HT}+$ OVX, $145.5 \pm 21.1 \mathrm{mg} ; \mathrm{BZA0.3,} 261.0 \pm 16.4 \mathrm{mg} ; \mathrm{BZA} 1$, $134.7 \pm 13.1 \mathrm{mg}$; mean $\pm \mathrm{SD}$; see Additional file 2: Figure S2]) The incidence of aneurysmal rupture and the body weight in BZA1 and HT rats were similar, suggesting that $1 \mathrm{mg} / \mathrm{kg}$ BZA revoked the effect of estrogen deficiency on the vasculature and the body weight without increasing the uterine weight.

\section{BZA upregulates ERa and ER $\beta$ expression in the cerebral artery}

As BZA is a SERM acting on ERs, we next investigated how treatment with BZA affected the ER expression in the cerebral artery of estrogen-deficient rats. Five weeks after bilateral renal ligation, we assessed the mRNA level and the protein expression of ERs in the left PCA where ruptured aneurysms had formed most frequently in experimental study 1.

$\mathrm{HT}+\mathrm{OVX}$ rats exhibited significantly lower mRNA levels of ER $\alpha$ and $E R \beta$ than did HT rats with intact ovaries. In BZA1 rats, the mRNA level of both ER $\alpha$ and ER $\beta$ was significantly elevated and was similar to the level in HT rats (Fig. 2a). In agreement with the mRNA level of ERs, ER $\alpha$, and ER $\beta$, protein expression was low in the cerebral arteries of $\mathrm{HT}+\mathrm{OVX}$ rats. In BZA1 rats, the expression of both ER $\alpha$ and ER $\beta$ was greater than in $\mathrm{HT}+\mathrm{OVX}$ rats (Fig. 2b). These findings indicate that BZA restored the gene and protein expression of both $E R \alpha$ and ER $\beta$ that was decreased by estrogen deficiency.
BZA downregulates IL-1 $\beta$ and decreases the MMP-9/TIMP-2 ratio in the cerebral artery

Elsewhere, we demonstrated that aneurysmal rupture in ovariectomized hypertensive rats was associated with the increase in the mRNA level of IL-1 $\beta$ and the MMP-9/ TIMP-2 ratio in the aneurysmal wall $[18,22]$. Here, we examined the mRNA level of IL-1 $\beta$, IL-6, TNF- $\alpha$, MMP-9, and TIMP-2 to explore the mechanisms underlying the protective effects of BZA against aneurysmal rupture.

The IL- $1 \beta$ mRNA level was significantly higher in HT + OVX than HT rats. In BZA1 rats, the level of IL-1 $\beta$ was significantly decreased and similar to the level in HT rats (Fig. 3a). The mRNA level of IL- 6 was similar among $\mathrm{HT}, \mathrm{HT}+\mathrm{OVX}$, and BZA1 rats (Fig. 3b). On the other hand, the mRNA level of TNF- $\alpha$ was significantly increased in $\mathrm{HT}+\mathrm{OVX}$ rats, and while it was lower in BZA1 rats, the difference did not reach statistical significance (Fig. 3c). These findings suggest that the gene expression of IL- $1 \beta$ and TNF- $\alpha$, but not of IL- 6 , is regulated at least partly by ERs.

The mRNA level of MMP-9 was significantly higher in $\mathrm{HT}+\mathrm{OVX}$ than HT rats. In contrast, the mRNA level of TIMP-2 was lower in $\mathrm{HT}+\mathrm{OVX}$ than $\mathrm{HT}$ rats. The mRNA level of MMP-9 in BZA1 rats was significantly lower, and TIMP-2 was higher than in HT+OVX rats (Fig. 4a, b). Consequently, the MMP-9/TIMP-2 ratio was reduced in BZA1 rats (Fig. 4c). These observations suggest that elevated MMP-9 mRNA- and attenuated TIMP-2 mRNA expression are associated with the downregulation of ERs. The downregulation of IL-1 $\beta$
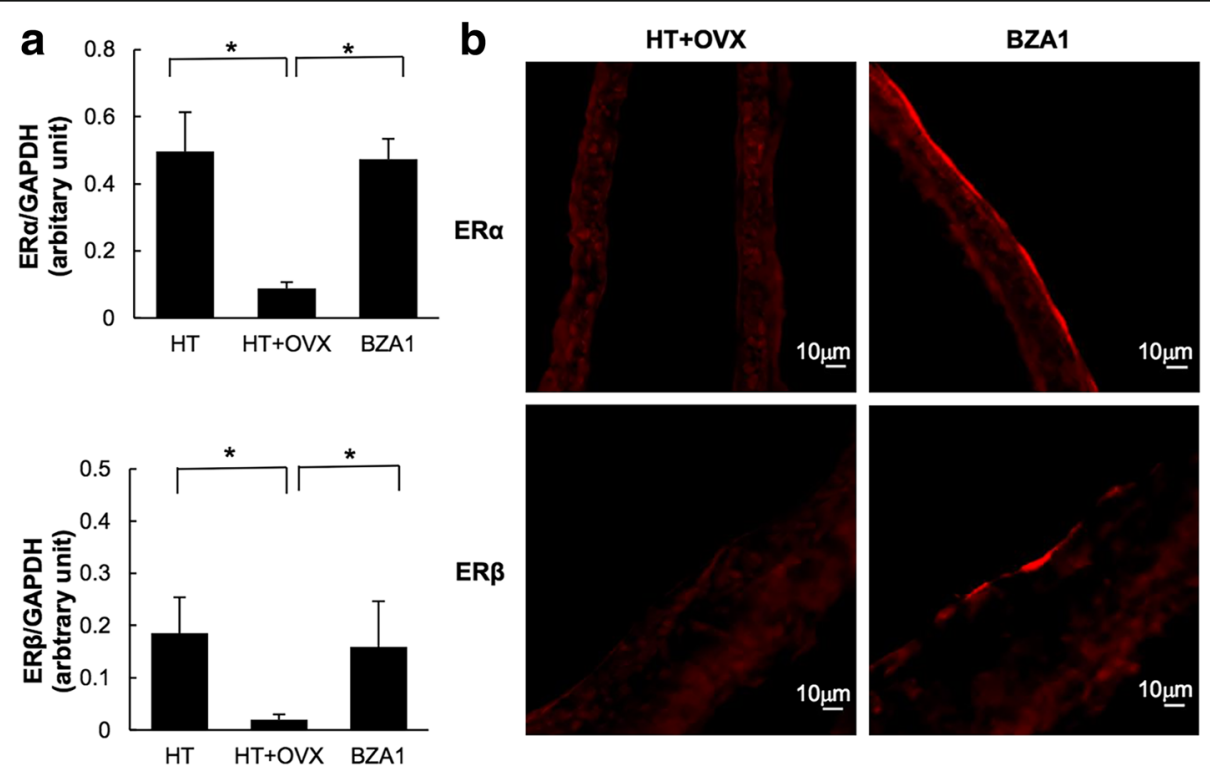

Fig. 2 a mRNA level of estrogen receptor (ER) $a$ and ER $\beta$ at the left posterior cerebral artery. The level was determined by quantitative real time-PCR and normalized by the glyceraldehyde 3-phophate dehydrogenase (GAPDH) mRNA level. $n=7$ per group, ${ }^{*} p<0.05$ (Scheffe's test). Data are the mean \pm SD. $\mathbf{b}$ Immunohistochemical staining for ERa and ER $\beta$ in the left posterior cerebral artery 

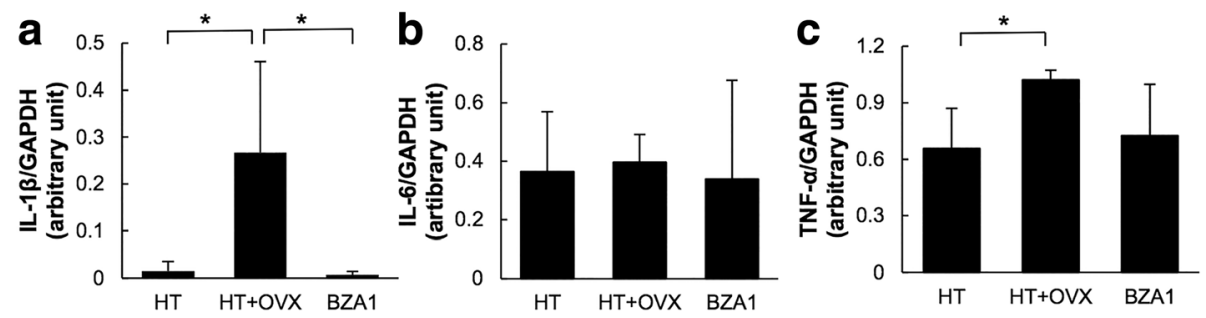

Fig. 3 mRNA level of $(\mathbf{a})$ interleukin (IL)-1 $\beta,(\mathbf{b}) I L-6,(\mathbf{c})$ tumor necrosis factor (TNF)-a in the left posterior cerebral artery. The levels were determined by quantitative real time-PCR and normalized by the glyceraldehyde 3-phophate dehydrogenase (GAPDH) mRNA level. * $p<0.05$ (Scheffe's test). Data are the mean \pm SD. $n=7$ per group

and the amelioration of the MMP/TIMP2 ratio via the modulation of ERs by BZA may have contributed to the prevention of aneurysmal rupture in our estrogendeficient hypertensive rats.

\section{Discussion}

We demonstrate that ovariectomy promoted aneurysmal rupture in hypertensive rats subjected to hemodynamic stress. We first document that in our hypertensive, ovariectomized aneurysm-model rats, the downregulation of ERs and the upregulation of pro-inflammatory molecules in the rupture-prone cerebral artery were associated with an increase in the aneurysmal rupture rate and that BZA ameliorated the downregulation of ERs, restored their gene and protein expression, decreased the incidence of aneurysmal rupture, and lowered the level of pro-inflammatory- and vascular degradation molecules. The incidence of ruptured aneurysms and the mRNA level of ERs and pro-inflammatory and vascular degradation molecules were similar to those in $\mathrm{HT}$ rats with intact ovaries. These findings suggest that BZA prevented aneurysmal rupture associated with inflammation and vascular degradation via the downregulation of ERs in our ovariectomized aneurysm-model rats.

Estrogen exerts diverse vascular effects mediated mainly by ER $\alpha$ and ER $\beta$ [23]. The treatment of ovariectomized rats with estradiol reversed the decrease in ER $\alpha$ and ER $\beta$ expression in the cerebral vessel wall [19]. Using our original aneurysm model, we demonstrated that estrogen deficiency elicited by ovariectomy reduced the expression of ER $\alpha$ while the expression of ER $\beta$ was retained at the bifurcation of the ACA-OA where unruptured aneurysms were highly and reproducibly induced [6]. The present study showed that the expression of ER $\alpha$ and ER $\beta$ in the PCA prone to aneurysmal rupture was reduced in our ovariectomized aneurysm rats and that BZA upregulated both ER $\alpha$ and ER $\beta$ in the PCA. Elsewhere, our group documented that in ovariectomized aneurysm mice, treatment with estrogen or an ER $\beta$-, but not an ER $\alpha$ agonist, reduced the incidence of cerebral aneurysm rupture [9]. The downregulation of ER $\beta$ is thought to be associated with inflammation [24]. We suggest now that in our current rat aneurysm model, BZA exerted protective effects against rupture through ER $\beta$ stimulation.

IL-1 $\beta$ promotes extracellular matrix degradation by increasing the production of MMPs and decreasing the production of TIMPs $[25,26]$. TNF- $\alpha$ and IL-6 also stimulate the expression of MMP-9 [27, 28]. In our current and earlier studies $[18,22]$, the increase in IL-1 $\beta$ and TNF- $\alpha$ and the imbalance between MMP-9 and TIMP-2 were associated with aneurysm rupture in ovariectomized aneurysm rats. BZA abated the increase in IL-1 $\beta$ and the imbalance between MMP-9 and TIMP-2 and resulted in a moderate inhibition of TNF- $\alpha$. ER $\beta$ stimulation decreased cellular inflammasome activity and IL-1 $\beta$ expression after global cerebral ischemia in
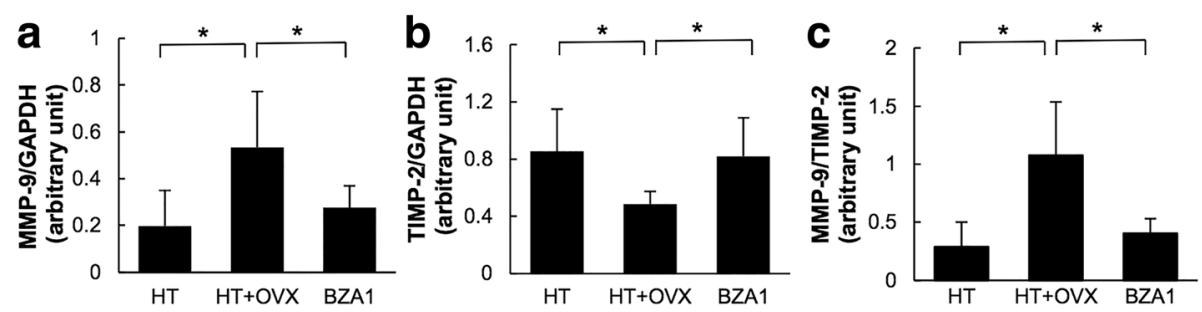

Fig. 4 mRNA level of (a) matrix metalloproteinase (MMP)-9 and (b) tissue inhibitor of metalloproteinase (TIMP)-2 in the left posterior cerebral artery. The levels were determined by quantitative real time-PCR and normalized by the glyceraldehyde 3-phophate dehydrogenase (GAPDH) mRNA level. c Ratio of the MMP-9/TIMP-2 mRNA level. * $p<0.05$ (Scheffe's test). Data represent the mean \pm SD. $n=7$ per group 
ovariectomized rats [24]. Selective ER $\beta$ agonists repress the transcription of pro-inflammatory genes including TNF- $\alpha$ [29]. The ER $\beta$ agonistic effects of BZA may be responsible for the downregulation of pro-inflammatory cytokines, resulting in the inhibition of vascular degradation.

Others reported that TNF- $\alpha$ modulates cerebral aneurysm formation and rupture. The TNF- $\alpha$ inhibitor 3,6 'dithiothalidomide resulted in aneurysmal stabilization and a significant decrease in the rupture rate [30]. In contrast, a meta-analysis reiterated that IL-6 was not associated with the pathogenesis of intracranial aneurysms [31]. These studies support our present findings.

In women, ER $\alpha$ activation in the brain stimulated physical activity and reduced their food intake and body weight gain [32, 33]. Therefore, the effect of BZA at $1 \mathrm{mg} / \mathrm{kg}$, but not $0.3 \mathrm{mg} / \mathrm{kg}$, on the body weight of our rats may have involved the upregulation of ER $\alpha$. On the other hand, since ER $\beta$ is thought to be associated with vasoprotection, the effect of BZA on the body weight via the upregulation of ER $\alpha$ may be smaller than the vasoprotective effects elicited by ER $\beta$. Estradiol and a selective ER $\alpha$ agonist increased the uterine weight in mice [15]. However, BZA had no such effects in the current and an earlier study [20], indicating that its effects are tissue-specific.

Use of our original rat aneurysm model resulted in a high incidence (more than 80\%) of unruptured aneurysms at the ACA-OA bifurcation; at most, $10 \%$ of these aneurysms ruptured during the 12-week observation period. For the current study, we modified the hemodynamics of our original model and produced a novel model in which approximately $50 \%$ of the experimentally induced aneurysms spontaneously ruptured [18]. With this new model, we demonstrated the protective effect of BZA, a selective ER modulator, on cerebral aneurysm rupture. The efficacy for prevention of aneurysm rupture may provide a new therapeutic option in the clinical setting.

Our study has some limitations. First, we assessed the changes in the mRNA level and the protein expression of the ERs elicited by ovariectomy and BZA. For a better understanding of the effects of individual ERs, studies using specific ER agonists or inhibitors and knockout animal are needed. Second, we started the administration of BZA 2 weeks after aneurysm induction without confirming the presence of cerebral aneurysms, and we did not confirm the presence of unruptured aneurysm in rupture-prone arteries at 12 weeks. Therefore, it is unclear whether BZA prevented their formation or their rupture. Third, the rodent age may affect their vascular response to estrogen [34], and menopause simulated by ovariectomy in our rats may be different from natural menopause in aged rats. Further studies are underway to address these questions.

\section{Conclusions}

This is the first study to show that BZA exerted protective effects against aneurysmal rupture in hypertensive ovariectomized aneurysm rats without affecting their blood pressure. It ameliorated the downregulation of ERs, the upregulation of IL-1 $\beta$, and the MMP-9/TIMP-2 imbalance in the cerebral artery. Since BZA has fewer estrogenic side effects than estrogen and is clinically used to address osteoporosis, it may help to prevent $\mathrm{SAH}$ in postmenopausal women.

\section{Additional files}

Additional file 1: Figure S1. A ruptured aneurysm in the left posterior cerebral artery (PCA) of an OVXNC rat (arrowhead). (TIFF $4425 \mathrm{~kb}$ )

Additional file 2: Figure S2. Wet uterine weight 5 weeks after aneurysm induction. ${ }^{*} p<0.05$ (Scheffe's test). Data represent the mean \pm SD. $n=7$ per group. (TIFF $2286 \mathrm{~kb}$ )

\section{Abbreviations}

ACA-OA: Anterior cerebral artery-ophthalmic artery; BZA: Bazedoxifene; ER: Estrogen receptor; GAPDH: Glyceraldehyde 3-phosphate dehydrogenase; HT: Hypertension; IL: Interleukin; MMP: Matrix metalloproteinase;

OVX: Ovariectomy; PCA: Posterior cerebral artery; qRT-PCR: Quantitative realtime PCR; SAH: Subarachnoid hemorrhage; SERM: Selective estrogen receptor modulator; TIMP-2: Tissue inhibitor of metalloproteinase-2; TNF: Tumor necrosis factor

\section{Acknowledgements}

Not applicable.

\section{Funding}

This work was supported by a Grant-in-Aid for Scientific Research [JSPS KAKENHI Grant Number JP15H04950], a Grant-in-Aid for Young Scientists (B) [JSPS KAKENHI Grant Number JP15K19972], and a Grant-in-Aid for the Strategic Young Researcher Overseas Visits Program for Accelerating Brain Circulation from the Japan Society for the Promotion of Science [JSPS KAKENHI Grant Number JPS2407].

\section{Availability of data and materials}

All data generated or analyzed during this study are included in this published article [and its supplementary information files].

\section{Authors' contributions}

HM, YT, KY, TM, and KTK contributed to the conception and design. HM, YT, $T M$, and KTK took part in the acquisition and analysis or interpretation of data. HM, YT, and KTK wrote the manuscript. YK, JS, TH, and SN supervised the study. All authors critically reviewed and approved the submitted version of the manuscript.

\section{Ethics approval}

All experiments and protocols were approved by the Ethics Committee of the Institute of Biomedical Sciences, Tokushima University Graduate School and conducted in accordance with the National Institutes of Health Guidelines for the Care and Use of Laboratory Animals. Experiments were reported according to the ARRIVE guidelines. Before all surgical procedures, the rats were anesthetized with isoflurane inhalation (induction 4\%, maintenance $2-2.5 \%$ in room air) and a local subcutaneous bupivacaine $(0.25 \%)$ injection. Euthanasia was performed with an anesthetic overdose (4\% isoflurane inhalation).

Consent for publication

Not applicable.

Competing interests

The authors declare that they have no competing interests. 


\section{Publisher's Note}

Springer Nature remains neutral with regard to jurisdictional claims in published maps and institutional affiliations.

\section{Author details}

'Department of Neurosurgery, Institute of Biomedical Sciences, Tokushima University Graduate School, 3-18-15 Kuramoto-cho, Tokushima 770-8503, Japan. ${ }^{2}$ Department of Anesthesia and Perioperative Care, University of California, San Francisco, 1001 Potrero Ave, SFGH 1, San Francisco, CA 94110, USA.

Received: 2 June 2017 Accepted: 18 September 2017

Published online: 02 October 2017

\section{References}

1. Ruan C, Long H, Sun H, He M, Yang K, Zhang H, Mao B. Endovascular coiling vs. surgical clipping for unruptured intracranial aneurysm: a metaanalysis. Br J Neurosurg. 2015;29:485-92.

2. de Rooij NK, Linn FH, van der Plas JA, Algra A, Rinkel GJ. Incidence of subarachnoid haemorrhage: a systematic review with emphasis on region, age, gender and time trends. J Neurol Neurosurg Psychiatry. 2007;78:1365-72.

3. Algra AM, Klijn CJ, Helmerhorst FM, Algra A, Rinkel GJ. Female risk factors for subarachnoid hemorrhage: a systematic review. Neurology. 2012;79:1230-6.

4. Jamous MA, Nagahiro S, Kitazato KT, Satomi J, Satoh K. Role of estrogen deficiency in the formation and progression of cerebral aneurysms. Part I: experimental study of the effect of oophorectomy in rats. J Neurosurg. 2005;103:1046-51.

5. Jamous MA, Nagahiro S, Kitazato KT, Tamura T, Kuwayama K, Satoh K. Role of estrogen deficiency in the formation and progression of cerebral aneurysms. Part II: experimental study of the effects of hormone replacement therapy in rats. J Neurosurg. 2005;103:1052-7.

6. Matsushita N, Kitazato KT, Tada Y, Sumiyoshi M, Shimada K, Yagi K, Kanematsu Y, Satomi J, Nagahiro S. Increase in body Nat/water ratio is associated with cerebral aneurysm formation in oophorectomized rats. Hypertension. 2012;60:1309-15.

7. Tamura T, Jamous MA, Kitazato KT, Yagi K, Tada Y, Uno M, Nagahiro S. Endothelial damage due to impaired nitric oxide bioavailability triggers cerebral aneurysm formation in female rats. J Hypertens. 2009;27:1284-92.

8. Tada Y, Kitazato KT, Tamura T, Yagi K, Shimada K, Kinouchi T, Satomi Nagahiro S. Role of mineralocorticoid receptor on experimental cerebral aneurysms in rats. Hypertension. 2009:54:552-7.

9. Tada Y, Wada K, Shimada K, Makino H, Liang El, Murakami S, Kudo M, Shikata F, Pena Silva RA, Kitazato KT, et al. Estrogen protects against intracranial aneurysm rupture in ovariectomized mice. Hypertension. 2014;63:1339-44.

10. Longstreth WT, Nelson LM, Koepsell TD, van Belle G. Subarachnoid hemorrhage and hormonal factors in women. A population-based casecontrol study. Ann Intern Med. 1994:121:168-73.

11. Feigin VL, Rinkel GJ, Lawes CM, Algra A, Bennett DA, van Gijn J, Anderson CS. Risk factors for subarachnoid hemorrhage: an updated systematic review of epidemiological studies. Stroke. 2005;36:2773-80.

12. Mhurchu CN, Anderson C, Jamrozik K, Hankey G, Dunbabin D. Hormonal factors and risk of aneurysmal subarachnoid hemorrhage: an international population-based, case-control study. Stroke. 2001:32:606-12.

13. Rossouw JE, Anderson GL, Prentice RL, LaCroix AZ, Kooperberg C, Stefanick ML, Jackson RD, Beresford SA, Howard BV, Johnson KC, et al. Risks and benefits of estrogen plus progestin in healthy postmenopausal women: principal results from the Women's Health Initiative randomized controlled trial. JAMA. 2002;288:321-33.

14. Anderson $G L$, Limacher $M$, Assaf AR, Bassford T, Beresford SA, Black $H$, Bonds D, Brunner R, Brzyski R, Caan B, et al. Effects of conjugated equine estrogen in postmenopausal women with hysterectomy: the Women's Health Initiative randomized controlled trial. JAMA. 2004:291:1701-12.

15. Tada Y, Makino H, Furukawa H, Shimada K, Wada K, Liang El, Murakami S, Kudo M, Kung DK, Hasan DM, et al. Roles of estrogen in the formation of intracranial aneurysms in ovariectomized female mice. Neurosurgery. 2014:75:690-5. discussion 695

16. Lonard DM, Smith CL. Molecular perspectives on selective estrogen receptor modulators (SERMs): progress in understanding their tissue-specific agonist and antagonist actions. Steroids. 2002;67:15-24.
17. Van Den Bemd GJ, Kuiper GG, Pols HA, Van Leeuwen JP. Distinct effects on the conformation of estrogen receptor alpha and beta by both the antiestrogens ICI 164,384 and ICI 182,780 leading to opposite effects on receptor stability. Biochem Biophys Res Commun. 1999:261:1-5.

18. Miyamoto T, Kung DK, Kitazato KT, Yagi K, Shimada K, Tada Y, Korai M, Kurashiki Y, Kinouchi T, Kanematsu Y, et al. Site-specific elevation of interleukin-1 beta and matrix metalloproteinase-9 in the Willis circle by hemodynamic changes is associated with rupture in a novel rat cerebral aneurysm model. [published online ahead of print Octorber 26, 2016]. J Cereb Blood Flow Metab. 2016;37:2795-805.

19. Sengupta P. The laboratory rat: relating its age with human's. Int J Prev Med. 2013;4:624-30.

20. Komm BS, Kharode YP, Bodine PV, Harris HA, Miller CP, Lyttle CR. Bazedoxifene acetate: a selective estrogen receptor modulator with improved selectivity. Endocrinology. 2005:146:3999-4008.

21. Makino H, Tada Y, Wada K, Liang El, Chang M, Mobashery S, Kanematsu Y, Kurihara C, Palova E, Kanematsu M, et al. Pharmacological stabilization of intracranial aneurysms in mice: a feasibility study. Stroke. 2012;43:2450-6.

22. Korai M, Kitazato KT, Tada Y, Miyamoto T, Shimada K, Matsushita N, Kanematsu Y, Satomi J, Hashimoto T, Nagahiro S. Hyperhomocysteinemia induced by excessive methionine intake promotes rupture of cerebral aneurysms in ovariectomized rats. J Neuroinflammation. 2016;13:165.

23. Miller VM, Duckles SP. Vascular actions of estrogens: functional implications. Pharmacol Rev, 2008:60:210-41.

24. de Rivero Vaccari JP, Patel HH, Brand FJ 3rd, Perez-Pinzon MA, Bramlett HM, Raval AP. Estrogen receptor beta signaling alters cellular inflammasomes activity after global cerebral ischemia in reproductively senescence female rats. J Neurochem. 2016;136:492-6.

25. Eberhardt W, Huwiler A, Beck KF, Walpen S, Pfeilschifter J. Amplification of L-1 beta-induced matrix metalloproteinase-9 expression by superoxide in rat glomerular mesangial cells is mediated by increased activities of NF-kappa B and activating protein-1 and involves activation of the mitogen-activated protein kinase pathways. J Immunol. 2000;165:5788-97.

26. Poljak M, Lim R, Barker G, Lappas M. Class I to III histone deacetylases differentially regulate inflammation-induced matrix metalloproteinase 9 expression in primary amnion cells. Reprod Sci. 2014;21:804-13.

27. Yao JS, Zhai W, Fan Y, Lawton MT, Barbaro NM, Young WL, Yang GY Interleukin-6 upregulates expression of KDR and stimulates proliferation of human cerebrovascular smooth muscle cells. J Cereb Blood Flow Metab. 2007:27:510-20.

28. Moon SK, Cha BY, Kim CH. ERK1/2 mediates TNF-alpha-induced matrix metalloproteinase-9 expression in human vascular smooth muscle cells via the regulation of NF-kappaB and AP-1: involvement of the ras dependent pathway. J Cell Physiol. 2004;198:417-27.

29. Cvoro A, Tatomer D, Tee MK, Zogovic T, Harris HA, Leitman DC. Selective estrogen receptor-beta agonists repress transcription of proinflammatory genes. J Immunol. 2008;180:630-6.

30. Starke RM, Chalouhi N, Jabbour PM, Tjoumakaris SI, Gonzalez LF, Rosenwasser RH, Wada K, Shimada K, Hasan DM, Greig NH, et al. Critical role of TNF-alpha in cerebral aneurysm formation and progression to rupture. J Neuroinflammation. 2014;11:77.

31. Sathyan S, Koshy LV, Srinivas L, Easwer HV, Premkumar S, Nair S, Bhattacharya RN, Alapatt JP, Banerjee M. Pathogenesis of intracrania aneurysm is mediated by proinflammatory cytokine TNFA and IFNG and through stochastic regulation of IL10 and TGFB1 by comorbid factors. J Neuroinflammation. 2015;12:135.

32. Xu P, Cao X, He Y, Zhu L, Yang Y, Saito K, Wang C, Yan X, Hinton AO Jr, Zou $F$, et al. Estrogen receptor-alpha in medial amygdala neurons regulates body weight. J Clin Invest. 2015:125:2861-76.

33. Eckel LA. The ovarian hormone estradiol plays a crucial role in the control of food intake in females. Physiol Behav. 2011:104:517-24.

34. Tarhouni K, Guihot AL, Vessieres E, Toutain B, Procaccio V, Grimaud L, Loufrani L, Lenfant F, Arnal JF, Henrion D. Determinants of flow-mediated outward remodeling in female rodents: respective roles of age, estrogens, and timing. Arterioscler Thromb Vasc Biol. 2014:34:1281-9. 